
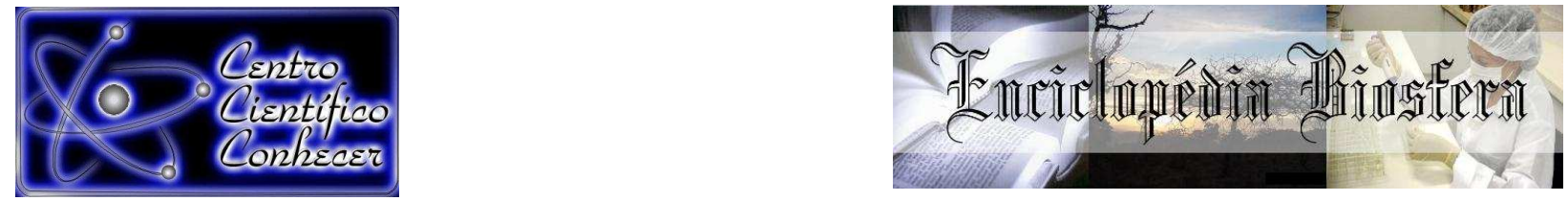

\title{
AVALIAÇÃO DA EXTRAÇÃO DE ÓLEO DE DENDÊ POR PRENSAGEM HIDRÁULICA NA COMUNIDADE DO LIMOEIRO, ACRE - BRASIL
}

Israel Silva de Souza ${ }^{1}$, Meireelem Santos Vieira ${ }^{2}$, Nadma Farias Kunrath ${ }^{1}$, Alcides Loureiro Santos ${ }^{1}$, Carlos Eduardo Garção de Carvalho ${ }^{2}$

1 Divisão de Tecnologia de Energia de Fontes Renováveis - DITER da Fundação de Tecnologia do Estado do Acre - FUNTAC (israel.silva@ac.gov.br) Rio Branco Brasil

2 Centro de Ciências Biológicas e da Natureza - CCBN da Universidade Federal do Acre - UFAC

Recebido em: 08/09/2015 - Aprovado em: 14/11/2015 - Publicado em: 01/12/2015 DOI: http://dx.doi.org/10.18677/Enciclopedia_Biosfera_2015_063

O presente trabalho teve por objetivo avaliar o rendimento da extração de óleo de palma, a partir de prensagem hidráulica manual, bem como a qualidade do óleo obtido no plantio de dendê (Elaeis guineensis) da comunidade do Limoeiro, em Rio Branco - Acre. A partir de uma amostragem de um universo de 107 palmeiras, foram coletados cachos maduros, dos quais os frutos foram submetidos à extração, para comparação de três ensaios: $E_{1}$ - sem cozimento prévio dos cachos; $E_{2}$ - com cozimento dos cachos em água a $80^{\circ} \mathrm{C}$ por 30 minutos e $\mathrm{E}_{3}$ - com cozimento dos cachos em água a $80^{\circ} \mathrm{C}$ por 60 minutos. Os experimentos foram feitos em triplicata e o rendimento foi calculado com base nas massas medidas. Para avaliar a qualidade do óleo, foram realizadas análises de massa específica, índice de acidez e saponificação. Em síntese, os resultados mostraram que o ensaio $\mathrm{E}_{2}$ gerou um maior rendimento de extração $(19,23 \%)$ e também um melhor índice de acidez $(3,00 \mathrm{mg}$ $\mathrm{KOH} / \mathrm{g}$ ), indicando a importância da realização do cozimento dos frutos, visto principalmente as grandes diferenças observadas entre os valores de índice de acidez dos óleos dos frutos sem cozimento quando comparados aos com cozimento. Pela metodologia adotada, foi possível alcançar resultados excelentes quando comparados a outros trabalhos reportados na literatura.

PALAVRAS-CHAVE: óleo de dendê, extração, índice de acidez

\section{CHARACTERIZATION AND EVALUATION OF PALM OIL OBTANEID BY HYDRAULIC PRESSING FROM LIMOEIRO COMMUNITY, ACRE - BRAZIL}

\begin{abstract}
The recent study aims to evaluate the palm oil yield of a sample of a universe of 170 palm trees (Elaeis guineensis) cultivated at small community known as Limoeiro, geographically located in proximity of Rio Branco capital the state of Acre. The oil was obtained by hydraulic extraction of the variety of mature clusters collected among the bunches. Afterwards the collected fruits then were exposed to different temperatures just before go through hydraulic extraction as following. $E_{1}-$ No
\end{abstract}


temperature was applied; $\mathrm{E}_{2}$ - clusters in water at temperature of $80^{\circ} \mathrm{C}$ for 30 minutes; $E_{3}$ - clusters in water at temperature of $80^{\circ} \mathrm{C}$ for 60 minutes. The extractions were performed by triplicates and the yield was calculated based on the measured masses. The quality of the palm oil was evaluated using the specific mass $(\mathrm{SM})$, acidity index ( $\mathrm{Al})$ and saponification index (SI). The results concluded that, best production efficiency and oil quality was obtained at $\mathrm{E}_{2}$ (yield $19.3 \%$ and $\mathrm{Al}=3.00$ $\mathrm{mg} \mathrm{KOH} / \mathrm{g}$ ), indicating the need of cooking the fruits at controlled temperature and time, despite of the results acquired from $E_{1}$ and $E_{3}$. Compared to previous research, according to literature, the results obtained from yielding capacity and oil quality at the community Limoeiro, reached satisfactory results.

KEYWORDS: palm oil, extraction, acidity index

\section{INTRODUÇÃO}

O dendezeiro (Elaeis guineensis Jacq.) é uma palmeira oleaginosa de origem africana que chegou ao Brasil através do tráfico negreiro, aproximadamente no século XVI. Os primeiros dendezais foram plantados no litoral da Bahia, onde o óleo de dendê passou a ser utilizado na culinária, disseminando o cultivo da oleaginosa em outras regiões do Brasil (ALMEIDA et al., 2013).

$\mathrm{Na}$ região Norte, o primeiro plantio em caráter comercial surgiu em 1968, no município de Benevides, no Pará, tornando-se, anos depois, o estado, um dos maiores produtores do país, por apresentar condições propícias ao desenvolvimento do cultivo (SUFRAMA, 2003).

A região amazônica apresenta o maior reservatório de biodiversidade do planeta, havendo a necessidade de conservação e uso de seu potencial de forma sustentável. Uma dessas opções é o uso de áreas degradadas, onde a ação antrópica é bastante presente. Nesse contexto, o dendê é uma alternativa, podendo proporcionar possibilidade de recobrimento dessas áreas, com associação de leguminosas que proporcionam o melhor uso do solo, ajudando a preservar o meio ambiente, além de poder gerar renda local com a comercialização do óleo e outros produtos (MÜLLER et al., 2006).

O estado do Acre possui uma área ainda pouco aproveitada no que se refere ao uso sustentável dos seus recursos, com diversas espécies de elevado potencial oleaginoso, como a copaíba, a andiroba, o cupuaçu, ururucum, buriti, murmuru, entre outras (KUNRATH et al., 2012).

Sobre o cultivo do dendê, o Acre ainda tem muito a desenvolver, possuindo pouca área plantada em propriedades particulares. As primeiras ações de pesquisa com o dendê no estado foram realizadas em 2006, em projetos relacionados com a geração de energia para comunidades isoladas, com a coordenação de Centro de Referência de Energia de Fontes Renováveis da Fundação de Tecnologia do Estado do Acre - FUNTAC, em parceria com a Embrapa. Foram adquiridas sementes comercias de quatro espécies (C2301, C2328, C2501 e C2528), provenientes do programa de melhoramento genético desenvolvido pela Embrapa, que foram inseridas em campos experimentais, cada um medindo 1,8 hectares, tanto no Vale do Juruá quanto no Vale do Acre. A área plantada totalizou aproximadamente 22 hectares, onde foi avaliado o estado nutricional da planta por meio de foliar, com acompanhamento da produção de cachos ano a ano (PEREIRA et al., 2010).

O óleo de palma (dendê) é o principal produto do dendezeiro, amplamente utilizado na indústria alimentícia, ocupando o primeiro lugar em produção mundial de óleos vegetais, com estimativa 2015/2016 de 65 milhões de toneladas, tendo a 
Indonésia e a Malásia como principais produtores (Producción Mundial de Aceite de Palma.com, 2015).

Além da indústria alimentícia, na qual é utilizado para fabricar manteiga, pães, biscoito, massas, tortas, azeite de dendê, e diversos outros produtos, o óleo de palma é usado ainda na produção de sabão, sabonetes, detergentes, velas aromáticas, tintas, fármacos, biodiesel, e outras aplicações (RSPO, 2013).

Apesar de existirem diferentes metodologias para extração de óleos, principalmente em escalas menores (laboratório), as escalas habituais para extração de óleo de palma estão no nível industrial. O processo de extração é baseado no esmagamento dos frutos, sendo que possui diversas etapas, que consistem basicamente no plantio, passando pela coleta dos cachos, esterilização, debulhamento, prensagem, desaeração, clarificação, decantação e armazenagem. Outro tipo de óleo é extraído a partir do esmagamento das amêndoas dos frutos, o óleo de palmiste, que é considerado um óleo mais nobre, aplicado na indústria de cosméticos (POKU, 2002).

A extração de óleos vegetais ocorre em todo território brasileiro, seja de forma industrial, semi-industrial, artesanal ou para fins de pesquisas básicas ou aplicadas. Particularmente, quando se trata das oleaginosas nativas da região Amazônica, normalmente as formas artesanais são adotadas, que estão presentes na cultura dos povos da floresta, sendo na realidade técnicas indígenas milenares, que em muitos casos trazem o sustento dessas famílias. Comumente nas comunidades, 0 óleo de dendê é extraído a partir da fervura dos frutos. Este método pode ser aplicado para vários tipos de frutos, principalmente os que contêm óleo no endocarpo, sendo utilizado para o buriti, tucumã, entre outros (CYMERYS et al., 2005).

Outra técnica de extração utilizada em comunidade é o da extração a frio ou quente através de prensa hidráulica manual, possibilitando maior rendimento de óleo quando comparado ao método da fervura em água. No Acre, essa técnica de extração faz parte da realidade das comunidades do Vale do Juruá, bem como as da Floresta Estadual do Antimary, as quais exploram espécies como o patauá, muito predominante na região (GOMES-SILVA et al., 2004).

Como no Acre a cultura do dendê e consequentemente a produção de óleo de palma é muito incipiente, o presente trabalho tem por objetivo avaliar a extração de óleo de palma de uma plantação cultivada em uma comunidade, bem como analisar a qualidade do óleo obtido, através de um processo de extração em baixa escala, utilizando uma prensa hidráulica manual, a fim de prospectar diferentes aplicações comerciais.

\section{MATERIAL E MÉTODOS}

\section{Localização, Amostragem e Coleta dos Cachos de Dendê}

As palmeiras estão localizadas na propriedade Silva e Silva, na comunidade do Limoeiro, em Rio Branco, próximo ao município de Porto Acre. O principal acesso até a comunidade é pela Rodovia Estadual AC-010 (Estrada do Quixadá), no km 19, entrando no ramal Boa Água, percorrendo mais $9 \mathrm{~km}$.

A propriedade foi adquirida em 1983 e sua área tem aproximadamente $4 \mathrm{~km}$ de largura de frente, $6 \mathrm{~km}$ de largura de fundo e $2 \mathrm{~km}$ de comprimento. Desde 2006, foram cultivadas 107 palmeiras de dendê na comunidade, em parceria com a EMBRAPA - ACRE e a Fundação de Tecnologia do Acre - FUNTAC. Quatro 
variedades comerciais foram plantadas, provenientes do programa de melhoramento genético da Embrapa Amazônia Ocidental: C2301, C2328, C2501 e C2528. O plantio foi realizado em covas de $40 \times 40 \times 40 \mathrm{~cm}$, no espaçamento de $9 \times 9 \mathrm{~m}$ em triângulo (7,8 m entre linhas e $9 \mathrm{~m}$ entre plantas na linha), sendo as linhas orientadas no sentido norte-sul.

Todas as palmeiras foram identificadas com plaquetas de alumínio, medindo aproximadamente $2,5 \times 4,5 \mathrm{~cm}$, com uma numeração para reconhecimento. Foi realizado um mapeamento mais detalhado de todo perímetro, georreferenciando cada palmeira através de um aparelho GPS, modelo MAP 76CSX, da marca GARMIN. A Figura 1 mostra o georreferenciamento das palmeiras estudadas.

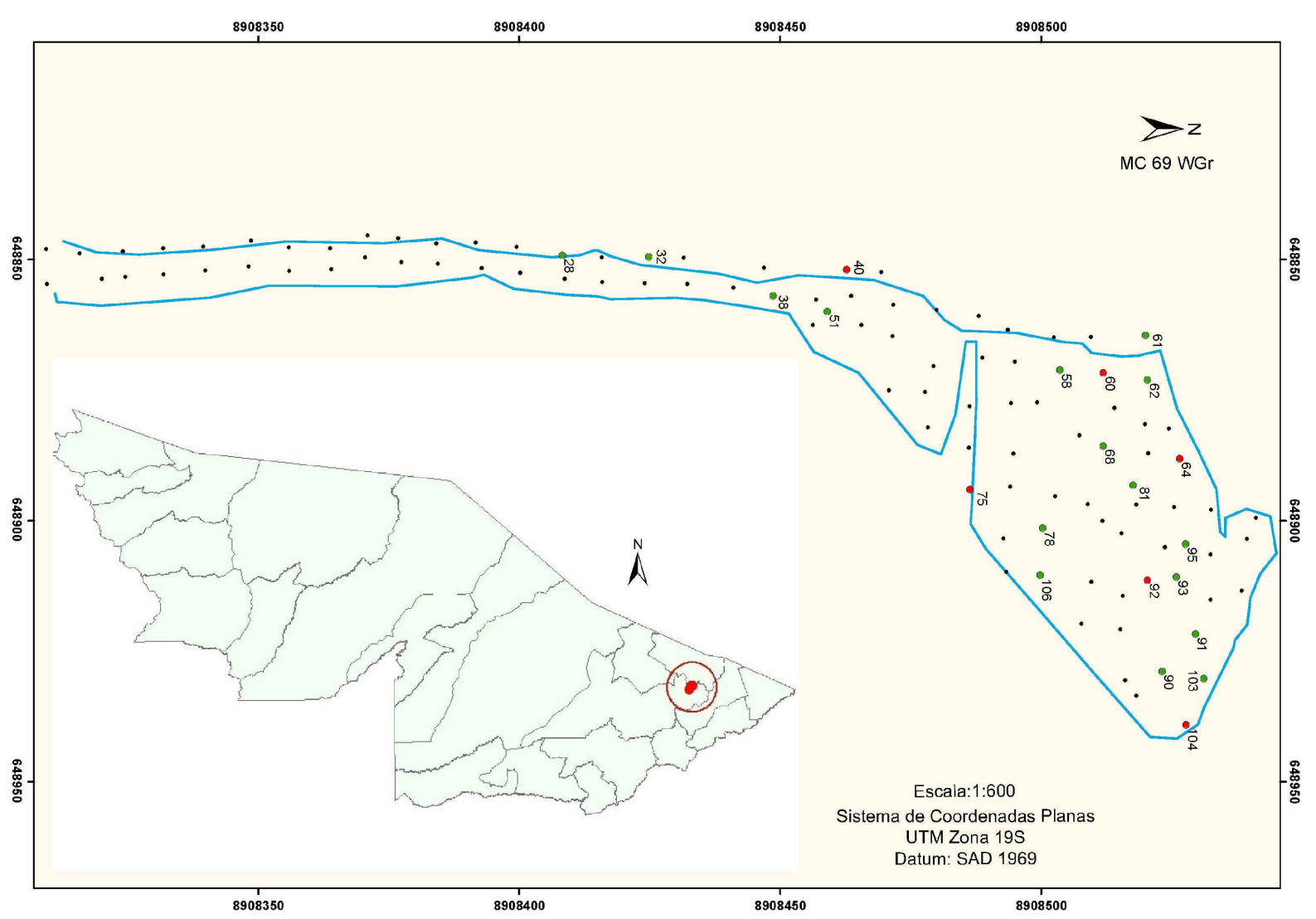

FIGURA 1: Georreferenciamento das palmeiras.

Fonte: Autores

Antes da coleta, foi realizada uma vistoria em toda área para identificar as palmeiras que continham cachos maduros. Foram identificadas, portanto, 22 plantas dispersas por todo o plantio, das quais foram escolhidas, por sorteio, 6 palmeiras para coleta, totalizando 10 cachos maduros. O Quadro 1 apresenta a identificação das palmeiras com cachos maduros, com destaque para as selecionadas para as extrações. 
QUADRO 1: Seleção de palmeiras de dendê para coleta de cachos maduros para extração de óleo

\begin{tabular}{|c|c|c|c|}
\hline Item & $\begin{array}{l}\text { Identificação } \\
\text { das Palmeiras }\end{array}$ & $\begin{array}{l}\text { Número } \\
\text { Total de } \\
\text { Cachos }\end{array}$ & $\begin{array}{c}\text { Número de Cachos } \\
\text { Maduros }\end{array}$ \\
\hline 1 & 28 & 5 & 1 \\
\hline 2 & 32 & 6 & 2 \\
\hline 3 & 38 & 5 & 1 \\
\hline 4 & 40 & 6 & 1 \\
\hline 5 & 51 & 6 & 2 \\
\hline 6 & 58 & 5 & 3 \\
\hline 7 & 60 & 7 & 1 \\
\hline 8 & 61 & 5 & 2 \\
\hline 9 & 62 & 6 & 1 \\
\hline 10 & 64 & 4 & 1 \\
\hline 11 & 68 & 5 & 2 \\
\hline 12 & 75 & 5 & 3 \\
\hline 13 & 78 & 6 & 1 \\
\hline 14 & 81 & 6 & 2 \\
\hline 15 & 90 & 6 & 1 \\
\hline 16 & 91 & 7 & 1 \\
\hline 17 & 92 & 5 & 3 \\
\hline 18 & 93 & 4 & 2 \\
\hline 19 & 95 & 5 & 2 \\
\hline 20 & 103 & 7 & 1 \\
\hline 21 & 104 & 6 & 1 \\
\hline 22 & 106 & 4 & 2 \\
\hline
\end{tabular}

A coleta dos cachos foi realizada de forma manual ou com a utilização de um facão, com cortes transversais na base, com o cuidado de não ferir ou perder os frutos. Os cachos foram armazenados em sacos de fibra e transportados até a sede da propriedade para o processamento.

\section{Extração do Óleo de Palma}

Para a extração do óleo de palma foi utilizada uma prensa hidráulica manual de capacidade até 15 toneladas de força, contendo um cilindro de aço com furo, fabricado especialmente para facilitar a saída de óleo (Figura 2). 


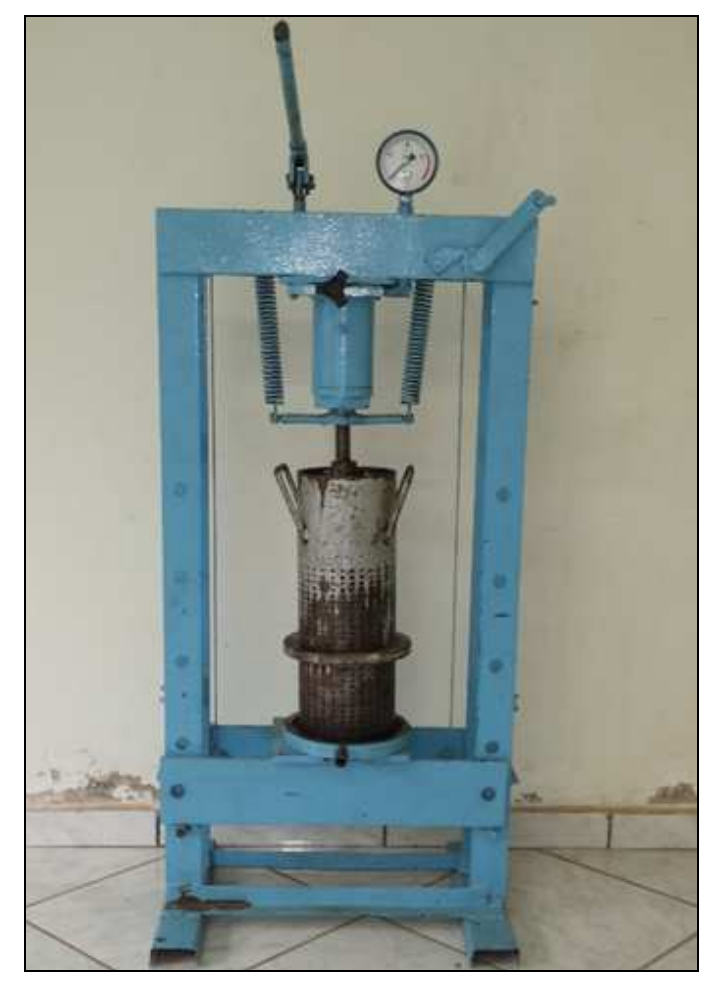

FIGURA 2: Prensa hidráulica manual utilizada para extração de óleo de palma Fonte: Autores .

Os ensaios de extração foram divididos em três partes: a primeira sem cozimento dos cachos $\left(E_{1}\right)$ e as duas seguintes com cozimento, em água quente, em dois tempos definidos, 30 minutos $\left(E_{2}\right)$ e 60 minutos $\left(E_{3}\right)$. O cozimento foi realizado em recipiente de ferro (tacho), com água aquecida a $80^{\circ} \mathrm{C}$. O aquecimento dos cachos tem como base o que é adotado em escala comercial de extração do óleo de palma, onde a água quente ou vapor d'água são aplicados para retirar o óleo a partir da ruptura das células oleosas dos frutos (MBA et al., 2015).

Os cachos foram debulhados com auxílio de um facão e foram preparadas três amostras $\left(R_{1}, R_{2}\right.$ e $\left.R_{3}\right)$, cada uma com aproximadamente $1 \mathrm{~kg}$ de frutos, pesadas em balança digital com exatidão de uma casa decimal do grama. Os frutos foram, então, submetidos à extração na prensa hidráulica. Para o cálculo do rendimento médio, foram medidas as massas dos óleos extraídos e comparadas às massas iniciais dos frutos que foram submetidos à extração.

Uma segunda etapa de extração foi realizada para cada torta obtida, separando-se as sementes, onde também foi medida a massa de óleo extraído, somando-se a da primeira etapa. Para estimar a qualidade dos óleos, foram realizadas as análises de índice de acidez, saponificação e massa específica, seguindo as normas do Instituto Adolfo Lutz (INSTITUTO ADOLFO LUTZ, 2008).

Para ilustração, a Figura 3 apresenta o fluxograma das etapas do processo de extração do óleo de palma. 


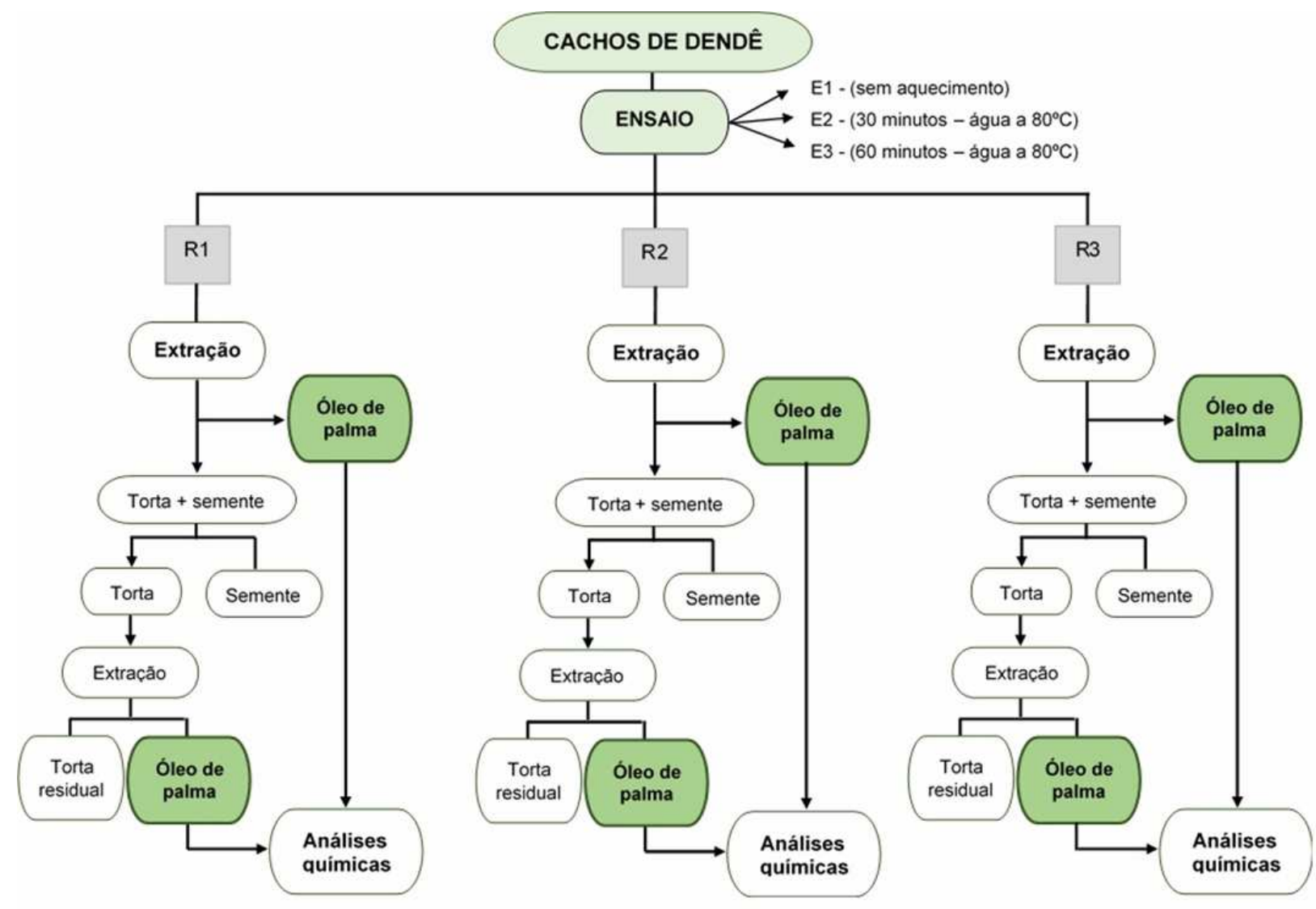

FIGURA 3: Fluxograma de extração do óleo de palma.

Fonte: Autores

\section{RESULTADOS E DISCUSSÃO}

Foi observado que $O$ aquecimento dos cachos nos ensaios $E_{2}$ e $E_{3}$ proporcionou uma maior facilidade de debulhamento dos frutos, os quais puderam ser separados de forma manual. Nos cachos que não sofreram cozimento $\left(E_{1}\right)$, foi necessário utilizar um facão para cortar o pedúnculo que liga os frutos do cacho, aumentando o tempo para realização dessa etapa.

A Tabela 1 apresenta os resultados de rendimento da extração de óleo de palma e das análises físico-químicas para os ensaios realizados.

TABELA 1: Resultados da etapa de extração de óleo de palma

\begin{tabular}{|c|c|c|c|}
\hline Ensaio & $E_{1}$ (sem cozimento) & $\mathrm{E}_{2}\left(30 \mathrm{~min} / 80^{\circ} \mathrm{C}\right)$ & $E_{3}\left(60 \mathrm{~min} / 80^{\circ} \mathrm{C}\right)$ \\
\hline Massa de óleo extraído $-1^{a}$ etapa $(\mathrm{g})^{*}$ & 134,27 & 135,23 & 134,33 \\
\hline Massa de óleo extraído $-2^{2}$ etapa $(\mathrm{g})^{\star \star}$ & 40,37 & 57,1 & 32,8 \\
\hline Rendimento médio de óleo $(\%)^{\star \star *}$ & 17,43 & 19,23 & 16,71 \\
\hline Índice de acidez médio (mg KOH/g) & 52,51 & 3,00 & 6,05 \\
\hline Índice de saponificação médio (mg KOH/g) & 207,13 & 193,52 & 204,55 \\
\hline Massa específica a $20^{\circ} \mathrm{C}\left(\mathrm{g} \cdot \mathrm{cm}^{-3}\right)$ & \multicolumn{3}{|c|}{0,888} \\
\hline
\end{tabular}

* Extração do óleo de palma dos frutos

** Extração do óleo da torta da primeira extração

*** Em relação à massa inicial dos frutos

ENCICLOPÉDIA BIOSFERA, Centro Científico Conhecer - Goiânia, v.11 n.22; p.3546 
Os rendimentos do óleo de dendê extraído nos diferentes ensaios foram próximos, sendo o ensaio $E_{2} \circ$ de maior valor, mostrando que o aquecimento dos cachos aparentemente não influenciou na quantidade de óleo extraído.

De acordo com POKU (2002), cozinhar os frutos enfraquece a estrutura da polpa, amaciando-a e tornando-se mais fácil para separar o material fibroso e os seus conteúdos durante o processo de digestão. A alta temperatura é suficiente para interromper parcialmente as células contendo óleo no mesocarpo e permite o óleo para ser liberado mais facilmente. Contudo, os resultados podem estar relacionados diretamente à força que foi aplicada na prensa (15 toneladas-força), que pode ter sido suficientemente alta para extrair a quantidade de óleo possível, estando os frutos amolecidos ou não.

É importante destacar que no primeiro esmagamento há a presença das sementes que, apesar de ser uma parte muito dura dos frutos, acaba limitando a força de extração, como cuidado para que as mesmas não sofram quebras. Normalmente nas indústrias, as sementes são separadas para a extração do óleo de palmiste de suas amêndoas, em prensas do tipo expeller (MBA et al., 2015).

Assim, torna-se importante a realização de uma segunda etapa de extração, da torta residual, para aumentar o rendimento em óleo de palma. Nesse trabalho, foi constatado que a quantidade de óleo da torta foi de $32,3 \%$, em média, em relação ao óleo extraído na primeira etapa, e de $42,2 \%$ somente para o ensaio $E_{2}$.

Outro ponto relevante foi a diferença da qualidade do óleo obtido, estimada pelos valores de índice de acidez, dos ensaios com aquecimento dos cachos em comparação ao sem "cozimento". O índice de acidez dos ensaios $E_{2}$ (3,00 mg $\mathrm{KOH} / \mathrm{g})$ e $\mathrm{E}_{3}(6,05 \mathrm{mg} \mathrm{KOH} / \mathrm{g})$ foram consideravelmente mais baixos do que o ensaio sem cozimento $(52,51 \mathrm{mg} \mathrm{KOH} / \mathrm{g})$. O óleo obtido pelo ensaio $E_{2}$ é considerado aceitável pelos padrões de qualidade exigidos para comercialização na indústria alimentícia, que sugerem estar até $4 \mathrm{mg}$ de $\mathrm{KOH} / \mathrm{g}$ (BRASIL, 2005). Além disso, tanto o óleo do ensaio E2 como do E3 possuem qualidade para outras aplicações, como produção de biodiesel (MEKHILEF et al., 2011). Isso ratifica, como em outros trabalhos, a importância do aquecimento dos cachos, para que além da facilidade de debulhamento dos frutos, façam a esterilização dos mesmos, eliminando os microorganismos que agem rapidamente na degradação do óleo de palma após colheita, produzindo ácidos graxos livres, além de outros subprodutos indesejáveis (LAM \& LEE, 2011).

É importante destacar que o ensaio $E_{2}$, além de apresentar um rendimento ligeiramente maior, gerou um óleo com índice de acidez igual à metade do obtido no experimento $E_{3}$, indicando em ambos os aspectos a não necessidade de uma etapa de aquecimento por mais de 30 minutos, nas condições adotadas. Com relação ao índice de saponificação, os valores apresentados estão de acordo com a literatura, que indica que o óleo de palma encontra-se na faixa de 190 a $209 \mathrm{mg}$ de $\mathrm{KOH} / \mathrm{g}$ (CHONG, 2012).

\section{CONCLUSÃO}

Pelas condições adotadas nesse trabalho, foi possível alcançar excelentes resultados de extração de óleo de dendê das espécies plantadas na Comunidade do Limoeiro, visto o rendimento e a qualidade do óleo extraído. Os resultados mostraram a importância da realização do cozimento dos cachos antes da extração, não necessitando ultrapassar de 30 minutos a $80^{\circ} \mathrm{C}$, o que permitiu uma maior 
facilidade de debulhamento dos frutos, maior rendimento em óleo e melhor índice de acidez. Apesar de ser um resultado bastante promissor, outros estudos devem ser realizados visando incentivar a comunidade para produção comercial.

\section{AGRADECIMENTOS}

À Comunidade do Limoeiro, em especial ao senhor Edson Rodrigues da Silva, pela autorização e apoio indispensável para a execução deste trabalho.

\section{REFERÊNCIAS}

ALMEIDA, D. T. D.; NUNES, I. L.; CONDE, P. L.; ROSA, R. P. S.; ROGÉRIO, W. F.; MACHADO, E. R. A quality assessment of crude palm oil marketed in Bahia, Brazil. Grasas y Aceites International Journal of Fats and Oils, vol. 64, n. 4, p. 387-394, jul/sept. 2013.

BRASIL. Agência Nacional de Vigilância Sanitária. Resolução RDC no 270, de 22 de setembro de 2005. Brasília, DF, 7 p. set. 2005.

CHONG, C. L. Measurement and maintenance of palm oil quality. In: LAI, O.-M.; TAN, C.-P.; AKOH, C. C. (Eds.). Palm oil: Production, processing, characterization and uses. Urbana, Illinois: AOCS Press, p. 433-470.2012.

CYMERYS, M.; FERNANDES, N. M. P.; RIGAMONTE-AZEVEDO, O.C. Buriti: Maurita flexuosa L. f. In: SHANLEY, P.; MEDINA, G. Frutíferas e plantas úteis na vida Amazônica. Centro para Pesquisa Florestal Internacional (CIFOR) e Instituto Homem e Meio Ambiente da Amazônia (IMAZON), Belém, p. 181-187.2005.

GOMES-SILVA, D. A. P.; WADT, L. H. de O.; EHRINGHAUS, C. Ecologia e manejo de patauá (Oenocarpus bataua mart.) para produção de frutos e óleo. Rio Branco, AC: Embrapa Acre, 2004. 37 p. (Documentos, 88).

INSTITUTO ADOLFO LUTZ. Métodos químicos e físicos para análise de alimentos. 4ํe․ São Paulo: IAL, 2008. 1020 p.

KUNRATH, N. F.; SOUZA, I. S. de; SANTOS, A. L. Caracterização dos resíduos provenientes do processo de extração do óleo do coco de murmurú (Astrocaryum spp) e seu potencial energético. In: Congresso Internacional de Bioenergia, 8, 2012, São Paulo. Anais. São Paulo: Congresso Internacional de Bioenergia, 2012. 1 CD ROM.

LAM, M.K.; LEE, K.T. Renewable and sustainable bioenergies production from palm oil mill effluent (POME): Win-win strategies toward better environmental protection. Elsevier. Biotechnology Advances. v. 29, p. 124-141. 2011.

MBA, O. I; DUMONT, M.-J.; NGADI, M. Palm oil: processing, characterization and utilization in the food industry - a review. Elsevier. Food Bioscience. v. 10. p. 2641. 2015. 
MEKHILEF, S.; SIGA, S.; SAIDUR, R. A review on palm oil biodiesel as a source of renewable fuel. Elsevier. Renewable and Sustainable Energy Reviews. v. 15, Issue 4, p. 1937-1949. 2011.

MÜLLER, A. A.; FURLAN JUNIOR, J.; CELESTINO FILHO, P. A Embrapa Amazônia Oriental e o agronegócio do dendê no Pará. Belém, PA: Embrapa Amazônia Oriental, 2006. 34 p. (Documentos, 257).

PEREIRA, J. B. M.; GUEDES, R. S.; ARAÚJO, E. A.; MASTRÂNGELO, J. P.; AMARAL, E. F.; OLIVEIRA, T. K.; OLIVEIRA T. K. DE; BARDALES, N. G. Combinação de cultivos florestais com a cultura da palma de óleo no estado do acre. In: RAMALHO FILHO, A.; Zoneamento agroecológico, produção e manejo da cultura de palma de óleo na Amazônia. Rio de Janeiro, RJ: Embrapa Solos, 2010. cap. 12 , p. 179-188.

POKU, K. Small-scale processing of oil palm in Africa. Rome: Food and Agriculture Organization of the United Nations, 2002. p. 7-19. (Agricultural Services Bulletin, 148).

Producción Mundial de Aceite de Palma.com. Aceite de Palma Producción Mundial 2015/2016. 2015.2 Disponível em: <www.produccionmundialaceitedepalma.com/> Acesso em: 13 de julho de 2015.

RSPO - ROUNDTABLE ON SUSTAINABLE PALM OIL. Como o óleo de palma está presente no seu dia-a-dia. Kuala Lumpur, 2013. 4 p.

SUFRAMA - SUPERINTENDÉNCIA DA ZONA FRANCA DE MANAUS. Potencialidades Regionais Estudo de Viabilidade Econômica. v. 5: Dendê, Manaus, 2003. 